\title{
Shaping Organic Microcrystals Using Focused Ion Beam Milling \\ Supporting Information
}

\author{
Wangxiang Li ${ }^{1}$, Jeremiah van Baren ${ }^{2}$, Adam Berges ${ }^{1}$, Elena Bekyarova ${ }^{1}$, Chun Hung Lui ${ }^{2}$, \\ Christopher J. Bardeen*1 \\ ${ }^{1}$ Department of Chemistry \\ University of California, Riverside \\ 501 Big Springs Road \\ Riverside, CA 92521 \\ 2 Department of Physics \\ University of California, Riverside \\ 900 University Ave. \\ Riverside, CA 92521

\section{*e-mail: christopher.bardeen@ucr.edu}

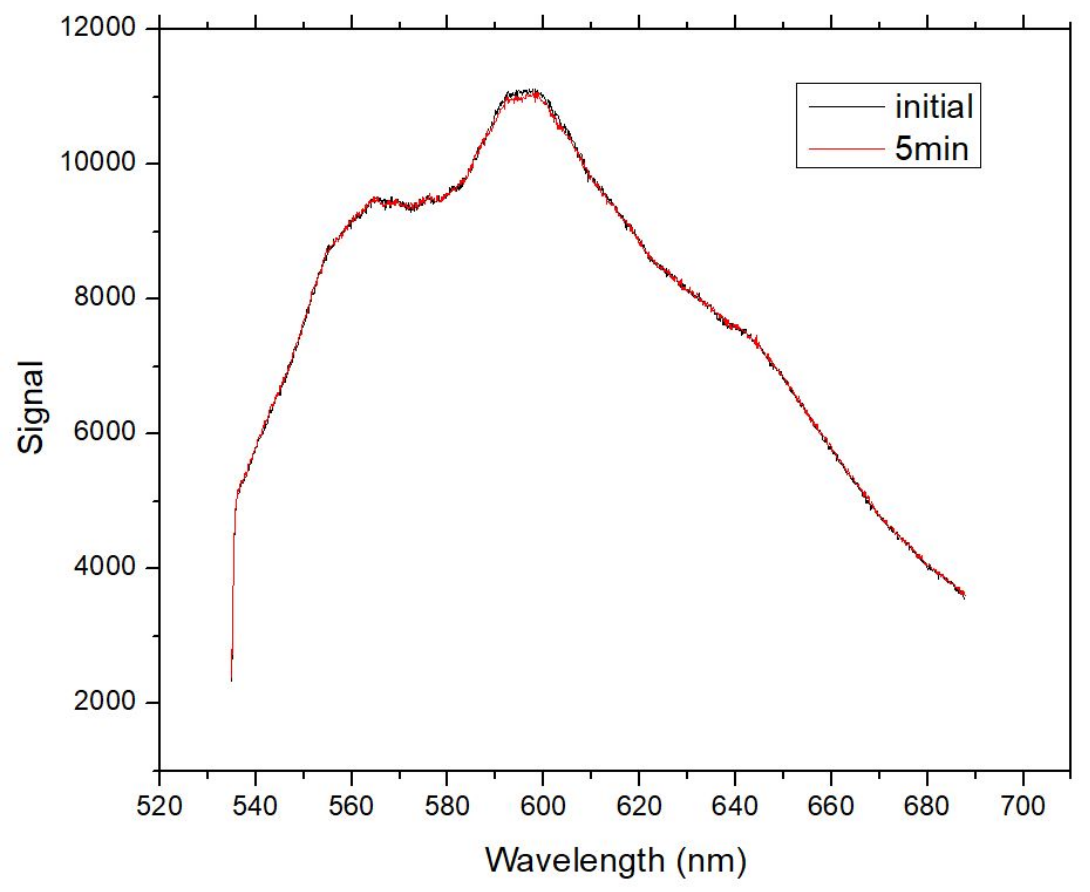

Figure S1. Photoluminescence intensity of the pure perylene crystal (without gold coating) before and after dipping into gold etchant. 


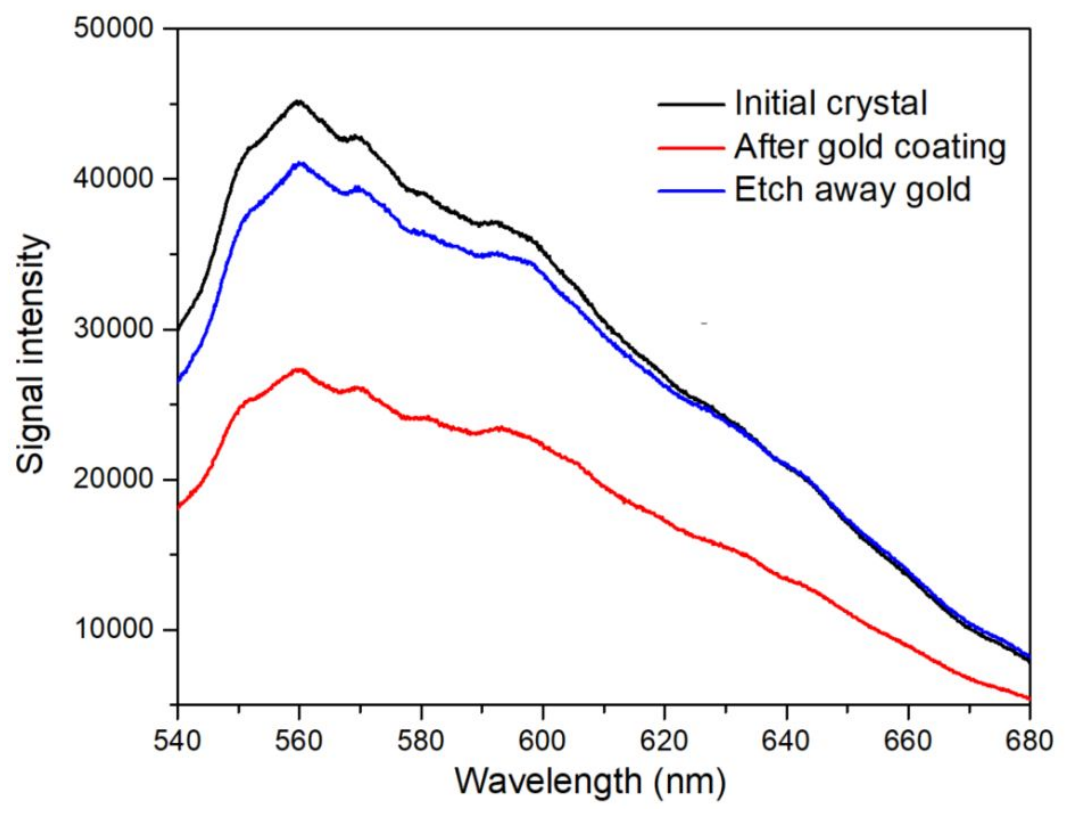

Figure S2. Photoluminescence intensity change of the Au-coated crystal without FIB exposure before and after the Au coating is etched away. This crystal was on the same substrate as a shaped crystal, but without direct exposure to ion beam.

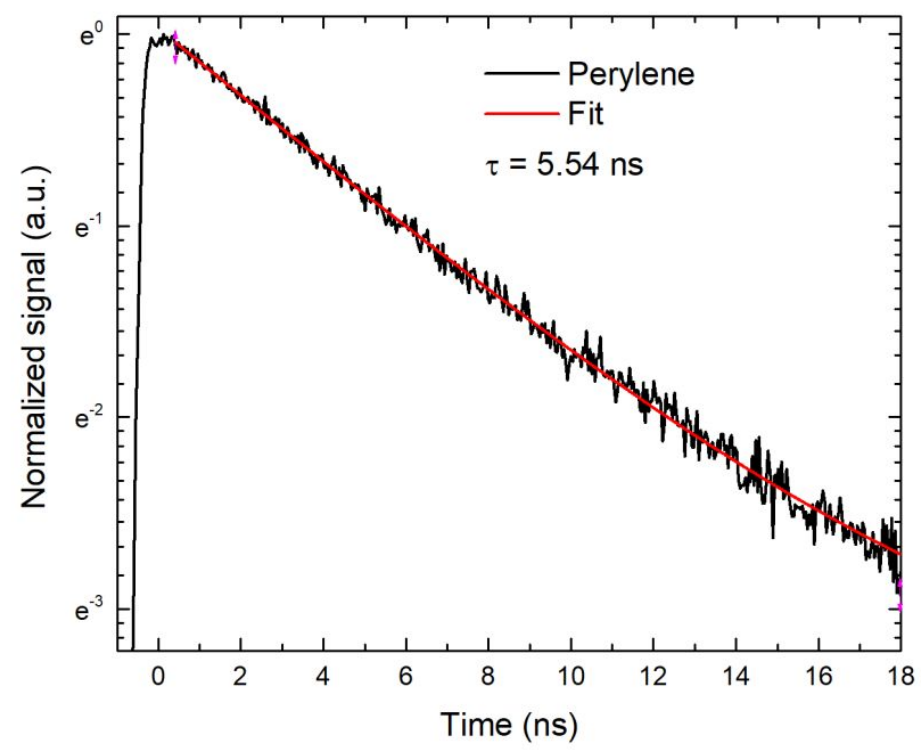

Figure S3. PL decays with biexponential fits of the original PER crystal excited by a $1 \mathrm{kHz}$ laser. The signal is collected by a streak camera. The average lifetime here is $5.54 \mathrm{~ns}$, which is longer than the result from single photon counting measurement using an $80 \mathrm{MHz}$ laser. 

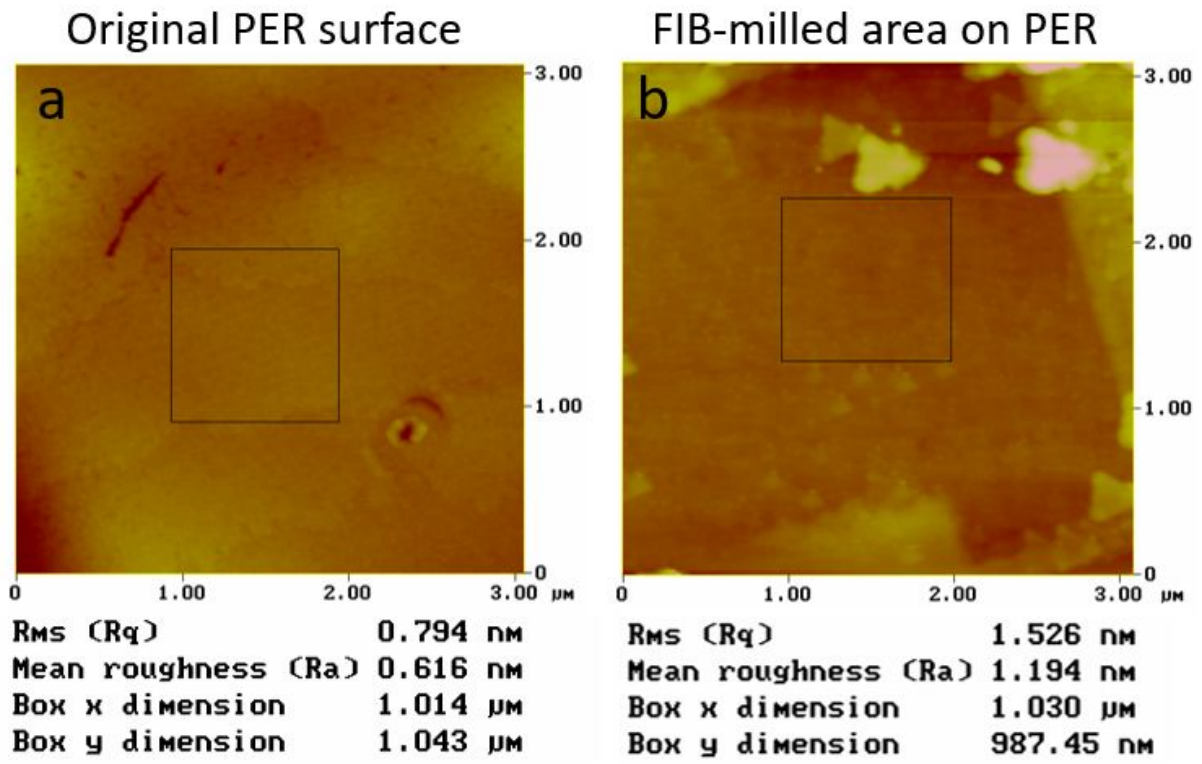

Figure S4. AFM roughness analysis of the Y-imprinted PER crystal (main text Figure 4) surface before (a) and after (b) FIB milling. The area in (b) is outside the Y shape, an area where the FIB beam removed material. The mean surface roughness increases from $0.6 \mathrm{~nm}$ to $1.2 \mathrm{~nm}$ after milling by the ion beam. 